\title{
Fortunato Santinis Bearbeitung von Carl Heinrich Grauns Der Tod Jesu als Beispiel der Pflege deutscher geistlicher Musik im Italien des frühen 19. Jahrhunderts
}

\author{
Andrea Pietro Ammendola
}

\section{Die zeitgenössische Beurteilung Santinis}

„,..., so ist Santini ein echter Sammler im besten Sinne des Worts ... Die Musik interessirt ihn eigentlich nicht viel, wenn sie nur in seinem Schranke steht; und er ist und hält sich für nichts, als einen ruhigen, fleißigen Arbeiter." ${ }^{\prime 1}$

Diese Beurteilung Felix Mendelssohn Bartholdys ist charakteristisch für die landläufige Rezeption des römischen Priesters und Musiksammlers Fortunato Santini (1778-1861). Betrachtet man zeitgenössische Äußerungen über Santini und dessen Sammlung näher, stellt man fest, dass die Meinungen deutlich auseinander gehen. So äußert sich beispielsweise Otto Nicolai in einem Brief an Georg Pölchau vom 18. September 1835²:

„Uebrigens ist er [Santini] auch ein wenig geldgierig und auch eitel: er sieht Baini mit schelen Augen an. Lassen wir ihn! ich stehe mit beiden gut, von Einem nehme ich Lehren - vom Anderen Noten! ... Dennoch ist es bei alledem schätzenswerth, sich eine Bibliothek aus Liebe zur guten Sache so gut man es verstanden hat erschrieben zu haben! Und in diesem Punkt ehre ich ihn."

Das Verhältnis zwischen Santini und dem von Nicolai erwähnten Giuseppe Baini (1775-1844) erschließt sich aus dem Reisetagebuch Carl Proskes. Der Eintrag vom 25. Oktober 1834 lautet $^{3}$ :

„Santini mit der Messe von Morales und der Überschrift: Baini cominciò, Santini continuò. - O italienische Mißgunst. Santini's Herzensergießung über die fruchtlose Bemühung, in die römischen Musikarchive zu gelangen. Äußerung desselben über Baini und wie die Römer ihm den Beinamen gegeben: il Cardinale del Papa di Prussia."

Einen Tag später besuchte Proske Baini, der "Santini's kopflose Tätigkeit“4 kritisierte. Santinis Klage über vergebliche Versuche, in römische Musikarchive zu gelangen, könnte damit zusammenhängen, dass Baini als Leiter der päpstlichen Kapelle seit 1814 mit der Reorganisation des päpstlichen Kapellarchivs betraut war. Andererseits lassen folgende Vermerke Santinis auf seinen

\footnotetext{
* Der vorliegende Beitrag ist eine stark verkürzte Fassung meiner Magisterarbeit (Münster 2005). Alle nachfolgenden mit SANT markierten Signaturen bezeichnen Handschriften und Drucke der Santini-Bestände in der Diözesanbibliothek Münster (D-MÜp). An dieser Stelle möchte ich den Bibliothekarinnen Barbara Glowka, Gertrud Gaukesbrink und Maria Zimmermann-Nonte für ihre Hilfsbereitschaft bei der Benutzung der Santini-Bestände danken.

1 Aus einem Brief Mendelssohns an seinen Vater vom 11.12.1830, in: Briefe aus den Jahren 1830 bis 1847 von Felix Mendelssohn Bartholdy, hrsg. v. P. Mendelssohn Bartholdy, Leipzig ${ }^{7} 1899$, S. 63.

2 Zitiert nach W. Virneisel, Otto Nicolai als Musiksammler, in: Festschrift Max Schneider zum achtzigsten Geburtstage, hrsg. v. W. Vetter, Leipzig 1955, S. 232 f.

${ }^{3}$ Fr. X. Haberl, Zum hundertsten Geburtstage von Dr. Karl Proske, in: Kirchenmusikalisches Jahrbuch 9 (1894), S. 36.

4 Ebd., S. 37.
} 
Manuskripten auf die wiederholte Unterstützung Bainis schließen: „favore D. Josephi Baini ${ }^{\prime 5}$, , ... Favore D. Josepho Baini sed Partitio facta fuit in domo sua ..." ${ }^{\prime 6}$. Santinis Autograph seines 1827 komponierten Te Deum enthält den Vermerk: ",corretted [sic] by Baini ${ }^{\prime 7}$. Demnach hat trotz aller Rivalität und dem Vorwurf einer "kopflose[n] Tätigkeit" auch Baini Santinis Arbeit zumindest im Rahmen der oben genannten Belege unterstützt.

Die zahlreichen positiven Stimmen zu Santini sollen freilich nicht unerwähnt bleiben, wie etwa in einem Beitrag des Musikschriftstellers Franz Sales Kandler $^{8}$ oder in der ersten Monographie über Santinis Sammlung von Wladimir Stassoff ${ }^{9}$. Es kann hier nur angedeutet werden, wie ambivalent die zeitgenössische Beurteilung Santinis war und sich geradezu polarisierend gestaltete; dabei ist der Einfluss Bainis auf das Meinungsbild nicht zu unterschätzen.

Anlass für den vorliegenden Beitrag ist die Tatsache, dass die Rezeption Santinis bis heute einseitig geblieben ist. Während seine Sammlung stets im Fokus der Betrachtung stand und steht, wurde Santini als Musiker erst im frühen 20. Jahrhundert etwa durch Friedrich Smend in den Vordergrund gestellt ${ }^{10}$. Bevor dieser Ansatz weiterverfolgt wird, lohnt ein kurzer Blick auf die Entstehungsgeschichte der Santini-Sammlung.

\section{Santini als Sammler}

Um 1800 begann Santini eine Sammlung vornehmlich kirchenmusikalischer Werke anzulegen, indem er aus Archiven und Bibliotheken Roms zahlreiches Notenmaterial italienischer Meister kopierte und spartierte. Der Komponist Giuseppe Jannacconi (1740-1816) ${ }^{11}$ hatte Santini musikalisch ausgebildet und ihn in die Kunst des Spartierens eingewiesen, weshalb dieser nach dem Tode Jannacconis 1816 einen Großteil von dessen Handschriften erbte ${ }^{12} .1820$ veranlasste ihn das bereits enorme Volumen seiner Sammlung zur Veröffentlichung eines Kataloges ${ }^{13}$, welche als Ausgangspunkt für eine grenzüberschreitende Bekanntheit Santinis im In- und Ausland zu markieren ist.

\footnotetext{
${ }^{5}$ SANT Hs 1702.

${ }^{6}$ SANT Hs 4282 .

${ }^{7}$ SANT Hs 3727.

${ }^{8}$ Fr. S. Kandler, Ueber den Musikzustand von Rom, in: Münchener Allgemeine Musikzeitung 1828, Nr. 30, Sp. $474 \mathrm{f}$.

${ }^{9}$ W. Stassoff, L'Abbé Santini et sa collection musicale à Rome, Florenz 1854.

${ }^{10}$ Fr. Smend, Zur Kenntnis des Musikers Fortunato Santini, in: Westfälische Studien. Beiträge zur Geschichte der Wissenschaft, Kunst und Literatur in Westfalen. Alois Bömer zum 60. Geburtstag gewidmet, Leipzig 1928, S. 90-98.

11 Jannacconi stand als Schüler und Erbe Pasquale Pisaris in der Tradition der Römischen Schule. Näheres bei L. M. Kantner, ",Aurea Luce“. Musik an St. Peter in Rom 1790-1850, Wien 1979 (= Veröffentlichungen der Kommission für Musikforschung 18), S. 54-56.

${ }^{12}$ Vgl. hierzu K. Kindler, Verzeichnis der musikalischen Werke Giuseppe Jannacconis (17401816) in der Santini-Sammlung in Münster (Westfalen), in: Fontes artis musicae 28 (1981), S. 313-319.

13 F. Santini, Catalogo della musica esistente presso Fortunato Santini in Roma: nel Palazzo de' Principi Odescalchi incontro la Chiesa de' SS. XII. Apostoli, Rom 1820, SANT Dr 1285.
} 
Santini ist es von Rom aus gelungen, im Laufe der Jahre ein Netzwerk an Auslandskontakten aufzubauen, ohne offenbar jemals selbst im Ausland zu Werbe- oder Tauschzwecken tätig gewesen zu sein. So sind aus England als wichtigste Tauschpartner Edward Goddard und Thomas Errington zu nennen, im französischsprachigen Raum waren es François-Joseph Fétis, Adrien de Lafage, Alexandre Choron und vor allem der Bibliothekar Auguste Bottée de Toulmon aus Paris ${ }^{14}$. Russische Musiksammler wie Alexander Skarjatin oder Stassoff sind ebenfalls als Tauschpartner und Abnehmer von Handschriften und Drucken belegt ${ }^{15}$.

Der Schwerpunkt der Santinischen Auslandsverbindungen lag jedoch im deutschsprachigen Raum. In Wien sind Aloys Fuchs und vor allem Raphael Georg Kiesewetter, aus Süddeutschland sind Anton Friedrich Justus Thibaut und der bereits erwähnte Proske anzuführen. Besonders rege - und für den vorliegenden Beitrag von entscheidender Bedeutung - waren Santinis Beziehungen zur Berliner Singakademie, namentlich zu Carl Friedrich Zelter, Felix Mendelssohn Bartholdy, Carl von Winterfeld und Otto Nicolai.

Durch diese multinationalen Verbindungen und durch den daraus resultierenden Tauschverkehr entstand eine der größten und vielseitigsten privaten Musiksammlungen des 19. Jahrhunderts ${ }^{16}$. Doch hat Santini kirchenmusikalische Werke nicht nur gesammelt, sondern darüber hinaus übersetzte Bearbeitungen ${ }^{17}$ angefertigt, um sie in Italien bekannt zu machen und Aufführungen zu ermöglichen. Eine Gelegenheit dazu bot sich durch seinen in der Mitte der 1830er Jahre in Rom gegründeten musikalischen Zirkel, der sich wöchentlich traf, um geistliche Musik aus seiner Sammlung kennenzulernen. Zu diesen Soi-

${ }^{14}$ Dass die genannten Personen als Tauschpartner fungierten, bezeugen zahlreiche Widmungen und Vermerke Santinis auf seinen Handschriften. Von Bottée de Toulmon ist außerdem ein Teil der Briefkorrespondenz erhalten und teilweise publiziert; vgl. hierzu Vl. Fédorov, A propos de quelques lettres de Santini à Bottée de Toulmon, in: Festschrift Karl Gustav Fellerer zum sechzigsten Geburtstag am 7. Juli 1962, hrsg. v. H. Hüschen, Regensburg 1962, S. 128-136.

15 Siehe dazu M. Janitzek, Santini - Stasov - Skarjatin. Drei Musiksammler, in: Festschrift für Winfried Kirsch zum 65. Geburtstag, hrsg. v. P. Ackermann u. a., Tutzing 1996 (= Frankfurter Beiträge zur Musikwissenschaft 24), S. 219-227.

${ }^{16}$ Die Sammlung umfasst zum jetzigen Zeitpunkt etwa 4500 Handschriften und etwa 1200 Drucke; vgl. den alphabetischen Katalog der Sammlung in drei maschinenschriftlichen Bänden von W. Wörmann, Münster o. J. (D-MÜms, D-MÜp). Die Bestände sind von RISM erfasst worden und bieten im Unterschied zu Wörmanns Katalog auch Nachweise über die Herkunft der Handschriften und Drucke. Von 1970 bis 1986 hat RISM einen Zettelkatalog der Santini-Sammlung erarbeitet, der heute als Teil der Reihe Musikhandschriften nach 1600 (Serie A/II) über CD-ROM und Internet zugänglich ist.

17 An dieser Stelle möchte ich auf ein terminologisches Problem hinweisen. Es erwies sich für die folgende Untersuchung als problematisch, sich auf einen der Termini Bearbeitung oder Übersetzung festzulegen. Santini operiert stets mit dem Begriff der Übersetzung (,tradotto", ",tra-

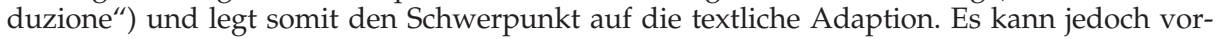
weggenommen werden, dass sich im Zuge der Analyse seiner italienischen Fassung von Carl Heinrich Grauns Der Tod Jesu auch musikalische Umgestaltungen zeigen. Daher wird Bearbeitung als allgemeiner Oberbegriff verwendet, Übersetzung mit Blick auf sprachliche Aspekte. 
reen kamen römische Dilettanten, Sänger der päpstlichen Kapelle und auswärtige Musiker. Stassoff und Alessandro Carcano berichteten über ein heute verschollenes Album, in das sich so prominente Namen wie Franz Liszt, Johann Baptist Cramer, François-Joseph Fétis und Fanny Mendelssohn eingetragen haben sollen ${ }^{18}$.

\section{Santini als Bearbeiter}

Santinis Interesse als Bearbeiter galt in erster Linie der deutschen Kirchenmusik, in geringerem Umfang der englischen. Dies bezeugen die zwei Sammelbände Composizioni sacre dall'Inglese in Latino tradotte da Fortunato Santini ${ }^{19}$ und Scelta di Musica antica dall'Inglese tradotta in Latino da Fortunato Santini ${ }^{20}$. Sie enthalten Werke etwa von Richard Farrant, Benjamin Rogers, Thomas Tallis und William Byrd. Das Hauptinteresse Santinis bezeugt wiederum Mendelssohn in einem Brief an Zelter vom 1. Dezember $1830^{21}$ :

„Er [Santini] nimmt sich vor, noch mehr deutsche Musik hier bekannt zu machen, ... Wenn Sie ihm bei Gelegenheit Manches von deutscher Musik könnten zukommen lassen, so wäre sein höchster Wunsch erfüllt; denn all' sein Sinnen und Trachten geht nun dahin, die deutsche Kirchenmusik in Italien einzuführen, und er besitzt von Seb. Bach bis jetzt nur die gedruckten Motetten und das Magnificat, von Graun nur das Te deum und die ,Passion'."

In der Tat fügt Santini dem Titel der meisten bearbeiteten Manuskripte Formulierungen wie „per favore conoscere il merito ${ }^{\text {"22 }}$ oder „per favore conoscere il bello"23 hinzu.

Schon länger ist bekannt, dass Santini zahlreiche geistliche Werke von Georg Friedrich Händel bearbeitet hat ${ }^{24}$, aber auch einige von Johann Sebastian Bach $^{25}$, Joseph Haydn ${ }^{26}$ und Wolfgang Amadeus Mozart ${ }^{27}$. Allerdings weiß man nicht, in welchem Umfang er auf ihre Aufführung über seinen römischen

${ }^{18}$ Vgl. Fédorov, V. V. Stasov chez l'abbé F. Santini à Rome, in: Anthony van Hoboken. Festschrift zum 75. Geburtstag, hrsg. v. J. Schmidt-Görg, Mainz 1962, S. 59; A. Carcano, Considerazioni sulla Musica Antica. Intitolate All'Ab. D. Fortunato Santini, Rom 1842, S. 6 f., Anm. 1.

19 SANT Hs 3616.

20 SANT Hs 3980.

${ }^{21}$ Briefe aus den Jahren 1830 bis 1847 von Felix Mendelssohn Bartholdy, S. 56.

22 Vgl. etwa SANT Hss 1883-1885.

${ }^{23}$ Vgl. SANT Hs 1875.

${ }^{24}$ Vgl. Näheres zu diesen Bearbeitungen bei K. G. Fellerer, Fortunato Santini als Sammler und Bearbeiter Händelscher Werke, in: Händel-Jahrbuch 2 (1929), S. 25-40; ferner R. Ewerhart, Die Händel-Handschriften der Santini-Bibliothek in Münster, in: ebd. 6 (1960), S. 111-150. In Betracht kommen die folgenden Handschriften: SANT Hss 1864, 1866, 1867, 1871, 1876, 1891, 1904.

25 Vgl. den in Anm. 10 erwähnten Aufsatz von Smend; außerdem Fellerer, Bachs Johannes-Passion in der lateinischen Fassung Fortunato Santinis, in: Festschrift Max Schneider zum achtzigsten Geburtstage, S. 139-145.

${ }^{26} \mathrm{Vgl}$. SANT Hs 2002.

27 Vgl. SANT Hss 2777, 2778, 3615. 
Zirkel hinaus einwirken konnte. Immerhin berichtet Mendelssohn aus Rom am 2. November 1830 an seine Familie ${ }^{28}$ :

„Das Erste von Musik, was ich hier sah, war ,der Tod Jesu' von Graun, den ein hiesiger Abbate, Fortunato Santini, recht gelungen und treu in's Italienische übersetzt hat. - Nun ist die Musik des Ketzers mit dieser Übersetzung nach Neapel geschickt worden, wo sie diesen Winter in einer großen Feierlichkeit ausgeführt werden soll, und die Musiker sollen ganz entzückt von der Musik sein und mit großer Liebe und Enthusiasmus an's Werk gehen."

Wenngleich nähere Einzelheiten zur erwähnten "großen Feierlichkeit" fehlen $^{29}$, bestätigt Stassoff, dass Santini einem Herzog de la Valle, Vorsteher der Società Filarmonica in Neapel, seine Bearbeitungen zum Zwecke der Aufführung zugeschickt habe ${ }^{30}$. Es handelt sich dabei wahrscheinlich um den neapolitanischen Patrizier Giuseppe della Valle, der am 9. September 1845 zum Verwaltungsrat der Königlichen Collegi di Musica San Sebastiano und San Pietro a Majella in Neapel ernannt wurde ${ }^{31}$.

\section{Carl Heinrich Grauns "Der Tod Jesu“ in der Bearbeitung Santinis}

\section{a. Quellen und Überlieferung}

Im Fokus der folgenden Betrachtung sollen Santinis Bearbeitungsmethoden stehen, sowohl mit Blick auf die Übersetzung als auch auf die sich daraus ergebenden Auswirkungen auf den Notentext. Hierfür erwies sich die von Mendelssohn erwähnte Bearbeitung von Carl Heinrich Grauns Der Tod Jesu als zweckmäßig und passend. Einerseits fehlen detaillierte Studien zu den italienischsprachigen Bearbeitungen Santinis nahezu ganz ${ }^{32}$, andererseits handelt es sich um eine vollständig überlieferte Bearbeitung eines größeren Werkes. Folg-

${ }^{28}$ Briefe aus den Jahren 1830 bis 1847 von Felix Mendelssohn Bartholdy, S. 38. In welchem Rahmen Mendelssohn diese Aufführung in Rom hörte ist unklar, möglicherweise in Santinis musikalischem Zirkel.

${ }^{29}$ Die erwähnte positive Rückmeldung geht aus dem bereits erwähnten Brief Mendelssohns an Zelter vom 1.12.1830 hervor (ebd., S. 56): „,er [Santini] ist es, der den ,Tod Jesu' übersetzt hat und in Neapel zur Aufführung bringt; in einem Brief, den er von dort erhalten hat, heißt es unter Anderm: ,Tutti i nostri dilettanti non vogliono udire adesso che musica di Graun et di Hendele; tanto è vero, che il bello non si puo perder mai. "“

30 Vgl. Stassoff, S. 26, Anm. 1.

${ }^{31}$ Vgl. L. F. Tagliavini, Johann Sebastian Bachs Musik in Italien im 18. und 19. Jahrhundert, in: Bachiana et alia musicologica. Festschrift Alfred Dürr zum 65. Geburtstag am 3. März 1983, hrsg. v. W. Rehm, Kassel u. a. 1983, S. 310 f., Anm. 48; vgl. ferner F. Florimo, La Scuola musicale di Napoli e i suoi Conservatori, Neapel 1880, Bd. 2, S. 151. Die endgültige Bestätigung findet sich in einem Eintrag eines gedruckten Kataloges des Conservatorio di musica di S. Pietro a Majella. Dort heißt es, dass Santini sein lateinisch übersetztes Jubilate Deo Händels einem „Sig G. De la Valle de' Duci di Ventignano" mit Widmung im Jahre 1838 schickte.

32 Die Wort-Ton-Relation der italienischen Bearbeitungen ist bisher nur marginal untersucht worden; vgl. Fellerer, Der Palestrinastil und seine Bedeutung in der vokalen Kirchenmusik des achtzehnten Jahrhunderts. Ein Beitrag zur Geschichte der Kirchenmusik in Italien und Deutschland, Augsburg 1929, S. 34 f. 
lich können die Bearbeitungsmethoden verschiedener Formteile eines geschlossenen Werkes untersucht werden ${ }^{33}$.

Zwei Quellen standen für die Auswertung zur Verfügung ${ }^{34}$.

Quelle A (siehe Abbildung 1) befindet sich in den Santini-Beständen der Münsterschen Diözesanbibliothek (Signatur SANT Hs 1735). Die Auswertung der insgesamt 14 Lagen lässt auf eine relativ uneinheitliche Anfertigung schließen. Gegen eine Reinschrift sprechen außerdem Verbesserungen im Sprach- und Notentext. Das Titelblatt der Handschrift besagt: La Morte di N.[ostro] S.[ignore] G.[esù] C.[risto] / Oratorio a 4. / di Carlo Enrico Graun / traduzione dal Tedesco di F.S. / la Poesia corretta da Luigi Bandelloni. Santini bezeichnet sich selbst explizit als Übersetzer und Luigi Bandelloni lediglich als Korrektor der Dichtung, weshalb Bandellonis Rolle über eine unterstützend-beratende nicht hinausgegangen sein dürfte ${ }^{35}$. Santinis Bearbeitung basiert auf einem

Abbildung 1: Quelle A, fol. $93^{\text {r }}$

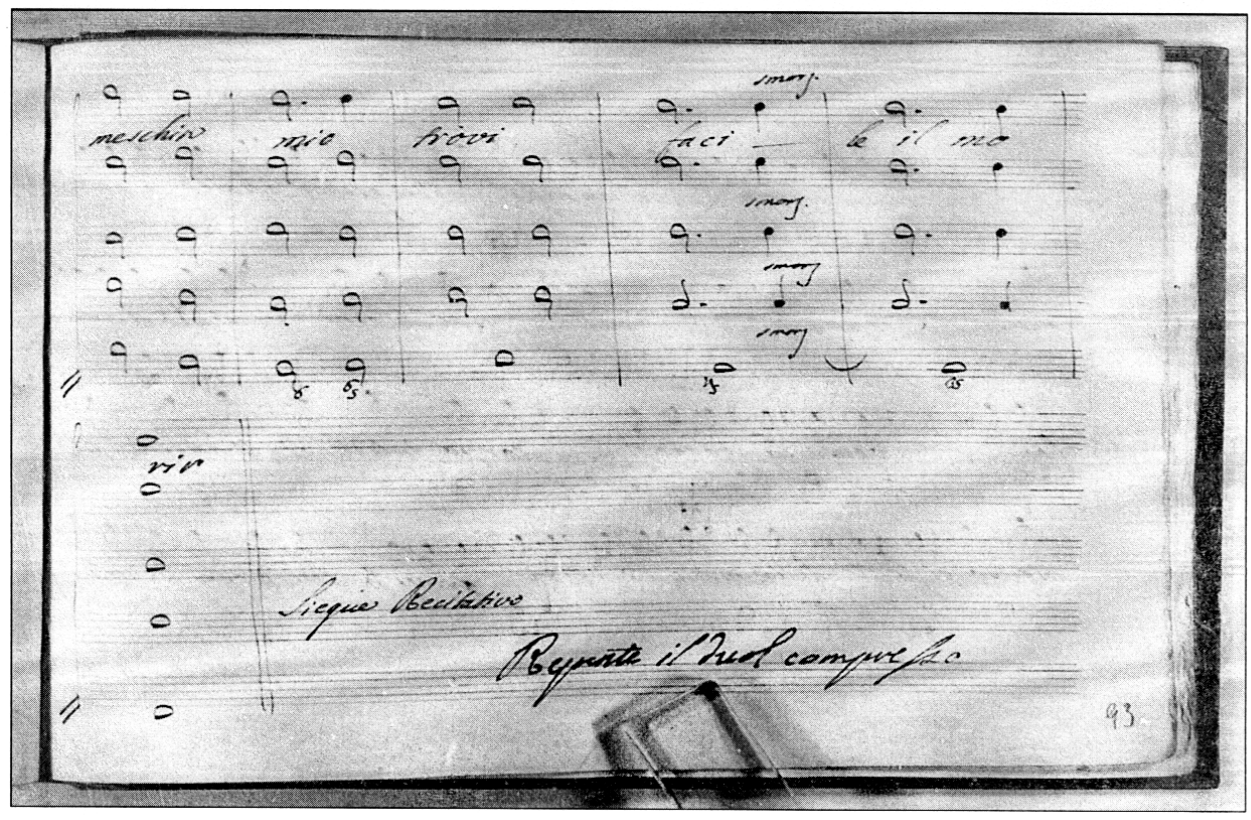

${ }^{33}$ Weitere Werke Grauns hat Santini offenbar nicht bearbeitet, obwohl ein halbes Dutzend Drucke und Abschriften Graunscher Kirchenmusik in Münster erhalten sind; vgl. Wörmann, Bd. 2, S. 243.

34 Der Vollständigkeit halber sei ein Fund in der Santini-Sammlung der Diözesanbibliothek Münster erwähnt, auch wenn er für diesen Beitrag inhaltlich keinen Mehrwert bringt. Es handelt sich um einen etwa DIN A5 großen Papierbogen aus einer dort separat aufbewahrten Loseblattsammlung, auf welchem kurze biographische Angaben zu Graun und Ramler notiert sind, die eindeutig von Santini stammen.

35 Luigi Bandelloni war ein römischer Dichter, Komponist und Zeitgenosse Santinis; vgl. Kandler, Ueber den Musikzustand von Rom, Sp. 455 f.; außerdem die sechs überlieferten Briefe Bandellonis an Kandler in Wien, Österreichische Nationalbibliothek, Handschriftensammlung, Signatur 6/27 (1-6). 
Druck mit dem Titel: Der / Tod Jesu / Kantate / in Musik gesetzt / von / C. H. Graun./ Partitur / Neue Ausgabe ${ }^{36}$. Ein Vermerk Santinis am oberen Rand des Titelblattes gibt folgenden Hinweis: „Fortunato Santini. Dono del Sig. Barone di Winterfeld -15. Aprile 1816".

Während eines zweijährigen Aufenthaltes von 1812 bis 1814 in Rom, Florenz und Venedig erforschte der Musikhistoriograph Carl von Winterfeld Werke italienischer Kirchenmusik und lernte währenddessen offenbar Santini kennen ${ }^{37}$. Der bei Stassoff zitierte Auszug aus einem Brief Winterfelds an Santini vom 12.3.1845 unterstreicht diese Vermutung ${ }^{38}$. Winterfeld erinnert dort recht nostalgisch an eine rund 30 Jahre zurückliegende Begegnung, bei der er mit Santini über gute Kirchenmusik philosophierte. Obgleich weitere Briefe nicht erhalten sind, belegen Santinis Eintragungen auf seinen Handschriften eine Korrespondenz mit Winterfeld während dessen Italienaufenthaltes und darüber hinaus ${ }^{39}$.

Winterfelds Bezug zu Grauns Der Tod Jesu ist eindeutig zu fassen. Er gehörte als Bass-Sänger von 1809 bis 1816 der Berliner Singakademie an, die den Tod Jesu bis weit ins 19. Jahrhundert jährlich am Karfreitag aufführte, so dass Winterfeld damit bestens vertraut gewesen sein muss ${ }^{40}$. Daher kann man davon ausgehen, dass Winterfeld Santini von der Graunschen Passion berichtete und möglicherweise versprach, ihm ein Exemplar aus Berlin zu schicken. In jedem Fall hat Santini am 15. April 1816 von Winterfeld die oben erwähnte Druckausgabe von Grauns Der Tod Jesu als Geschenk („dono“) erhalten. Mit Blick auf Santinis Bearbeitung lässt sich anhand der vorhandenen Quellen lediglich ein grob gefasster Zeitrahmen stecken. Durch Santinis Notiz „15. Aprile 1816“ ist zumindest der Terminus post quem ermittelbar. Endgültig fertiggestellt war die Bearbeitung spätestens im Laufe des Jahres 1830, wie der oben zitierte Brief Mendelssohns vom 2.11.1830 beweist.

Quelle B (Signatur SA 57, siehe Abbildung 2), bis vor kurzem unbekannt, wird in den Beständen der Berliner Singakademie als Depositum der Berliner Staatsbibliothek aufbewahrt. 2001 wurde sie als Teil der verschollenen Bestände der Singakademie von Kiew nach Berlin zurückgeführt und bereits auf Mikrofiche publiziert ${ }^{41}$. Die homogene Lagenordnung, das saubere Schriftbild und das folgende Titelblatt lassen auf eine als Präsentationsexemplar konzipierte Reinschrift schließen: La morte di Gesù / Cantata / di C. F. [sic] Graun / dal

${ }^{36}$ SANT Dr 339. Es handelt sich um ein Exemplar der dritten Druckausgabe (Breitkopf \& Härtel 1810).

37 Vgl. zu Leben und Werk Winterfelds B. Stockmann, Carl von Winterfeld. Ein Beitrag zur Geschichte der Musikhistoriographie im neunzehnten Jahrhundert, phil. Diss. Kiel 1957.

${ }^{38}$ Vgl. Stassoff, S. 14, Anm. 1.

39 SANT Hs 844: „Ex Barone de Winterfeld dum Romae degebat“; SANT Hs 872 (Nr. 1-10): „Ex Barone de Winterfeld / dum Florentiae degebat“; SANT Hs 4008 (Nr. 29): „Cosi publicato dal Barone di Winterfeld / Lipsia 1843".

${ }^{40} \mathrm{Vgl}$. Stockmann, S. 13.

${ }^{41}$ Musikhandschriften der Staatsbibliothek zu Berlin, Preußischer Kulturbesitz, Teil 6: Die Sammlung der Sing-Akademie zu Berlin, Teil 1: Oratorien, Messen, Kantaten und andere geistliche Werke, hrsg. von der Sing-Akademie zu Berlin u. a. 2004, Mikrofiche Nr. 043-046. 
Abbildung 2: Quelle B,Titelseite

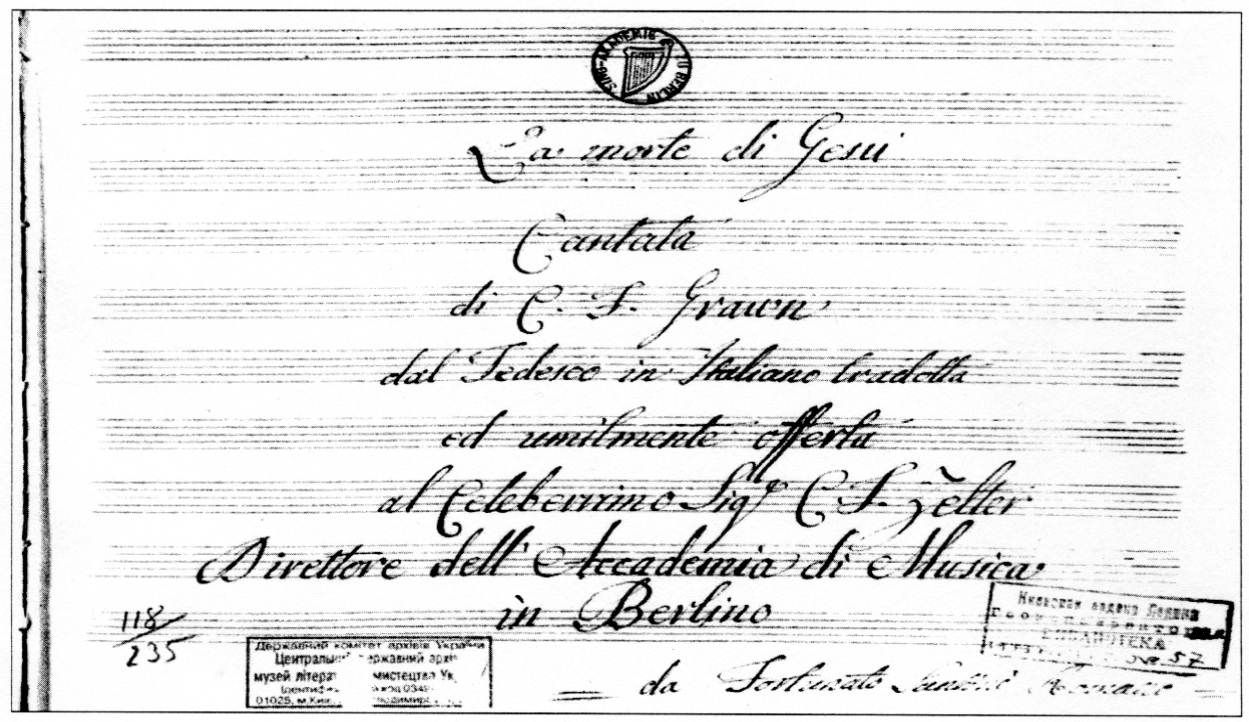

Tedesco in Italiano tradotta / ed umilmente offerta / al Celeberrimo Sig. C. F. Zelter / Direttore dell'Accademia di Musica / in Berlino / da Fortunato Santini Romano.

Die Überlieferung lässt sich wie folgt rekonstruieren: Aus dem bereits zitierten Brief Mendelssohns an Zelter vom 1.12.1830 geht hervor, dass Santini Zelter einige Handschriften - laut Mendelssohn „,vier dicke Bände, meistens Palestrina'sche Musik enthaltend ${ }^{\prime 42}$ - zukommen lassen wollte. In den Beständen der Singakademie in Berlin sind außerdem zwei Briefe Santinis an Zelter erhalten (vom 2.12.1830 und 24.2.1831), in welchen er die Zusendung der Abschriften ankündigt, darunter auch die Übersetzung von Grauns Der Tod Jesu $u^{43}$. Wann genau Zelter das Exemplar erhalten hat, ist nicht belegt. Im zweiten Brief Santinis vom 24.2. heißt es, dass er Zelter die Übersetzung ein anderes Mal (,,in altra occasione") zuschicken werde. Fertiggestellt hat Santini seine Reinschrift bereits Ende 1830, was die letzte Seite der Quelle (fol. 119) beweist: „Laus Deo, Beatae Virgini Mariae; ac Santae Ceciliae / die 20 Decembris 1830“. Demnach ist Santinis Präsentationsexemplar vermutlich im Laufe des Jahres 1831 in die Bestände der Singakademie gelangt.

Ein Vergleich der beiden beschriebenen Quellen führt zu dem Ergebnis, dass sie inhaltlich-musikalisch kaum differieren. Man kann daher davon ausgehen,

42 Briefe aus den Jahren 1830 bis 1847 von Felix Mendelssohn Bartholdy, S. 56.

${ }^{43}$ Diese zwei Briefe sind einer Motettensammlung (Palestrina, Allegri, Victoria und Nanino) vorgeheftet, die ebenfalls Zelter gewidmet ist (D-B SA 413). 
dass die Anfertigung der Reinschrift für Zelter auf der Basis von Quelle A geschah, welche noch eindeutige Spuren einer Konzeptfindung erkennen lässt.

\section{b. Die italienische Übersetzung}

Auf welche Art und Weise hat Santini Karl Wilhelm Ramlers Passionsdichtung ins Italienische übersetzt? Mendelssohns oben zitierte Bemerkung, der Text sei ,recht gelungen und treu ins Italienische übersetzt ${ }^{\prime \prime 4}$ soll im Folgenden konkretisiert werden. Zunächst ist zu beobachten, dass sich Santini zwischen einer - mit Blick auf Ramlers Dichtung - nachahmenden und einer eigenständigen Reimform bewegt. Um Santinis Übersetzung näher zu kommen, bietet sich eine dreifache Kategorisierung $\mathrm{an}^{45}$.

Die erste Kategorie umfasst die wörtlichen Übersetzungen. Darunter fallen Übersetzungen, die syntaktisch mit dem deutschen Text übereinstimmen (1 und 2), syntaktisch nicht übereinstimmen (3 und 4) und sich durch leichte $\mathrm{Ab}$ weichungen mit Blick auf die Wortwahl auszeichnen (5 und 6):

\section{Rezitativ Nr. 6}

Betrachtend steht der Menschenfreund und spricht,

Pensoso sta' dell'uom' l'amico e dice

2 Der Geist ist willig, nur der Leib ist schwach;

Lo spirto è pronto si, ma il corpo è infermo,

3 Rezitativ Nr. 8

Ich kenne diesen Menschen nicht.

Io quest'uom non conosco.

4 Arie Nr. 13

So steht der Held aus Canaan.

Si l'Eroe di Canaa stà.

5 Choral Nr. 21

die Gott den Frommen vorbehält.

che pei giusti serba Iddio.

[die Gott für die Gerechten bewahrt.]

6 Arie Nr. 7

theilt die Wolken

fende i venti

[teilt die Winde]

Insgesamt ist zu beobachten, dass wörtliche Übersetzungen gehäuft in den Rezitativen und seltener in den Arien, Chorälen und Chören vorkommen. Der erzählende Sprachstil der Ramlerschen Rezitative kommt dieser Übersetzungsform eher entgegen als etwa die stärker bildhaften Arien. Die von mir un-

\footnotetext{
44 Briefe aus den Jahren 1830 bis 1847 von Felix Mendelssohn Bartholdy, S. 38.

45 Die deutschen Beispiele folgen der Textunterlegung im oben erwähnten Graunschen Partiturdruck von 1810, die Satznummern entsprechen der Abfolge in Grauns Vertonung.
} 
ternommene und in eckige Klammern gesetzte wörtliche Rückübersetzung in den beiden letzten Beispielen (5 und 6) zeigt, dass der Inhalt trotz geringer Unterschiede nahezu gleich bleibt. Übersetzungen des letzten Typus erscheinen anteilig häufiger als die exakt-wörtlichen, da diese keine sprachlichen Freiheiten in der Übertragung zulassen.

Die zweite Kategorie bilden die sinngemäßen Übersetzungen. Diese entspringen nicht unmittelbar der deutschen Vorlage, sondern übertragen den ursprünglichen Bedeutungsgehalt in einer sprachlich freieren Form:

\section{Choral Nr. 5}

Wer nimmt sich meiner Seelen an, wenn nun mein Leben nichts mehr kann, Chi avverrà sostegno sia alla debil alma mia?

8 Arie Nr. 4 [Wer wird meiner schwachen Seele Halt geben?]

Du Held, auf den die Köcher des Todes ausgeleert, Tu ch'hai,..., il telo franto a morte,

9 Chor Nr. 25 [Du, der ..., den am Tode zerbrochenen Pfeil inne hat.]

mit Thränen diesen Staub zu netzen, der deine Lebensbäche trank, Di stille spargiamo la polve beata che tutta inaffiata dal tuo sangue fu, [In Tränen zerstreuen wir den seligen Staub, der gänzlich von deinem Blut benetzt war.]

Die wörtlichen und sinngemäßen Übersetzungen sind sich insofern ähnlich, als beide Typen hinsichtlich ihrer Bedeutungsübertragung relativ eng am deutschen Text bleiben. Der Unterschied besteht im Grad der sprachlich-exakten Übersetzung. Vor allem die gezeigten metaphorischen Adaptionen in 8 und 9 belegen das Bemühen Santinis um eine möglichst nahe Übertragung.

In die dritte Kategorie fallen modifizierende Übersetzungen. Auffällig sind zunächst Ausdrücke, die bei Ramler als typisch empfindsame Wendungen wie etwa der Begriff ",heiter" - vorkommen und sich bei Santini selbst dann zeigen, wenn sie im deutschen Text nicht gesetzt sind. Das Adjektiv "dolce" (süß, sanft, angenehm) hebt sich dabei besonders hervor:

\section{Choral Nr. 5}

Bist du es, Gott, mein Heiland, nicht?

mi darai dolce aita o Signor.

11 Rezitativ Nr. 8

[wirst du mir süße Hilfe geben, o Herr.]

Doch siehe! Jesus wendet sich,

Eppur Gesù dolce si volge

[Und doch wendet sich Jesus sanft.]

Darüber hinaus ist Santinis Sprache von zahlreichen Ausschmückungen und emphatischen Zusätzen durchsetzt, um die göttliche Macht durch den Lobpreis des Menschen oder das Leiden Jesu hervorzuheben: 
12 Chor Nr. 25

Anbetung sey dein Dank, den opfre jedermann.

l'uomo con dolci modi offra bei serti e lodi, cor grato e pura fe.

[Der Mensch soll auf angenehme Arten schöne Kränze und Lobpreisungen,

13 Choral Nr. 24 ein dankbares Herz und lauter Glauben opfern.]
Konnte seine sanfte Brust so viel Schmerz ertragen?
Il suo gentil frale dovea mai soffrire tant'onte, tant'ire, tant'aspro martor.
[Seine anmutige Brust durfte niemals soviel Schande, soviel Zorn, soviel raue Qual erleiden.]

Weitere Zusätze dieser Art zeigen sich etwa in Choral Nr. 24, in welchem Jesus sein Leben "unter unendlicher Qual" (,fra pena infinita") verlassen muss. In Rezitativ Nr. 3 ruft er "unter Schmerzen und laut" (,in duolo e forte") und legt in Rezitativ Nr. 22 am Kreuz seinen Kopf "in solchem Schmerz" (,in tal dolore“) auf die Brust. In Chor Nr. 10 ist das "schmerzhafte Herz in Tränen" (,il cor dolente in pianto") ",demüthig" (,umile") zu Boden gebeugt, während die reumütigen Sünder in Chor Nr. 25 mit "unendlichen Vergehen" (,falli infiniti") belastet sind.

Man kann als Ergebnis dieser dreiteiligen Kategorisierung festhalten, dass Santini grundsätzlich darum bemüht ist, die Nähe zum Ramlerschen Text zu wahren. Dies belegen einerseits die zahlreichen wörtlichen Übersetzungen, welche vorwiegend in Rezitativen zu finden sind. Santinis Anlehnung an den deutschen Text zeigt sich andererseits an der großen Anzahl der sinngemäßen Übersetzungen. Den ersten beiden Kategorien steht die dritte der modifizierenden Übersetzungen gegenüber. Neben den typisch empfindsamen Zusätzen - es sei an die frequente Verwendung des Wortes "dolce" erinnert - zeichnet sich diese Kategorie vor allem durch eine ausgeschmückte und affektbetonte Sprache aus.

Es stellt sich abschließend die Frage, inwieweit Santini in seiner Übersetzung den neologisch-empfindsamen Tendenzen des Ramlerschen Librettos ${ }^{46}$ gefolgt ist. Auffällig ist zunächst, dass Santini die Bezeichnungen für Jesus, die bei Ramler auf einem stark anthropologisch geprägten Jesusbild basieren, übernimmt: "L'amico ai mortal" („Freund der Sterblichen", Choral Nr. 24), „L'amico dell'uomo" („Menschenfreund", Choral Nr. 24, Rezitativ Nr. 6) und "Il meglior d'ogni mortale" ("Der Beste unter den Sterblichen", Rezitativ Nr. 3). Darüber hinaus belegen einige Textstellen, dass Santini die bei Ramler kaum akzentuierte göttliche Natur Jesu einflicht. In Chor Nr. 25 findet sich für Jesus die Bezeichnung "göttlicher Herr" („divin Signor"), in Arie Nr. 4 zeichnet sich Jesus durch

\footnotetext{
${ }^{46}$ Vgl. hierzu ausführlich H. Lölkes, Ramlers „,Der Tod Jesu” in den Vertonungen von Graun und Telemann. Kontext - Werkgestalt - Rezeption, Kassel u. a. 1999 (= Marburger Beiträge zur Musikwissenschaft 8), besonders S. 51-83; ferner I. König, Studien zum Libretto des „Tod Jesu“ von Karl Wilhelm Ramler und Karl Heinrich Graun, München 1972 (= Schriften zur Musik 21).
} 
seine "göttliche Macht" („,divin possente ${ }^{\prime)}$ aus, und in Rezitativ Nr. 22 legt er sein Haupt auf seine "göttliche Brust" (,divin petto"). Die Verlagerung auf die menschliche Natur Jesu ist demnach bei Santini nicht so eindeutig wie bei Ramler.

Eine Textstelle ist besonders bemerkenswert: Statt des vierten Kreuzwortes Jesu „Mein Gott, mein Gott, warum hast du mich verlassen?"48 erscheinen bei Ramler in Rezitativ Nr. 22 diese Worte modifiziert: „Mein Gott! Mein Gott! Wie hast du mich verlassen!" Nach Herbert Lölkes gibt Ramler die Worte "nicht als Frage wieder, die an den Verstand appelliert, sondern als Ausruf, der sich primär an das Gefühl wendet ${ }^{\prime 49}$. Bei Santini wurde diese Modifikation nicht übernommen, sondern traditionell als Frage formuliert: „O Padre mio, perché m'abbandoni?" [O Vater, warum verlässt du mich?]. Fraglich ist, ob Santini diese empfindsame Modifizierung Ramlers bewusst nicht übernommen hat oder die genannten traditionell-italienischen Kreuzesworte in Frageform unreflektiert einsetzte.

Zugunsten einer explizit katholisch-dogmatischen Gewichtung hätte Santini den Ramlerschen Text trotz der genannten Beispiele stärker modifizieren müssen. Der lyrische und affektbetonte Sprachgestus Ramlers wird von Santini äußerst treu nachgezeichnet und durch eine emotional stärkere Sprache vielmehr noch gesteigert.

\section{c. Die musikalische Bearbeitung}

Santini folgt in der musikalischen Anlage dem Graunschen Druck: Er übernimmt sämtliche Schlüssel und behält auch die Satzfolge exakt bei. Unisonoführungen in den Instrumentalstimmen notiert Santini nicht doppelt, sondern verweist auf die ausgeschriebene Stimme.

Bezogen auf die Prosodie des Textes lässt sich allgemein feststellen, dass Santini grundsätzlich bemüht ist, den Graunschen Notentext möglichst zu übernehmen. Dies gelingt sehr oft nur mit Vokalverschleifungen, wenn der italienische Text mehr Silben aufweist als der deutsche, wie zu Beginn des Anfangschorals:

$\begin{array}{llllllll}14 & 1 & 2 & 3 & 4 & 5 & 6 & 7\end{array}$

$\mathrm{Du}$, des-sen Au-gen flos-sen

$$
\begin{array}{cccccccc}
1 & 2 & 3 & 4 & 5 & 6 & 7 & 8 \\
\text { Chi puo fre-na-re } & \text { il pian-to }
\end{array}
$$

In diesem Fall ermöglicht die Vokalverschleifung von (fre-na-)re mit il

$$
\begin{array}{cccccccc}
15 & 1 & 2 & 3 & 4 & 5 & 6 & 7 \\
\multicolumn{4}{c}{\text { Chi puo fre-na-re il pian-to }}
\end{array}
$$

\footnotetext{
${ }^{48}$ Mt 27,46 und Mk 15,34 nach Ps 22,2.

49 Lölkes, S. 61.
} 
eine exakte Übertragung des Graunschen Notentextes. In vielen Fällen jedoch ist eine solche Verschleifung nicht möglich, so dass Santini gezwungen ist, Notenwerte aufzuteilen bzw. zusammenzuziehen. Die Singstimmen-Melodie wird in diesen Fällen dem italienischen Text angepasst, während der ursprüngliche Notenwert in den Instrumentalstimmen zumeist erhalten bleibt.

Santinis Bearbeitung der Choräle zeichnet sich größtenteils durch textbedingte Änderungen aus. Neben zahlreichen Verschleifungen fallen vor allem die aufgelösten Halbe-Auftakte der einzelnen Choralzeilen auf. Bis auf Choral Nr. 1 werden in allen übrigen Chorälen die ursprünglich auftaktigen Halbenoten zu Vierteln aufgelöst, um die Deklamation des italienischen Textes aufrecht zu erhalten. Hinsichtlich des Graunschen Notentextes ergibt sich daraus ein veränderter Sprachduktus, der den gleichmäßigen, in Halben fortschreitenden Charakter der Choräle verändert und ein erhöhtes Deklamationstempo zur Folge hat.

Neben diesen sprachlich bedingten Anpassungen greift Santini auch aus Gründen der Ausdruckssteigerung in die Graunsche Vorlage ein. Am markantesten zeigt sich dies im Schlussabschnitt des Chorals Nr. 21 Wie herrlich ist die neue Welt (siehe Notenbeispiel 1a und b). Hier augmentiert Santini die Takte 25 und 29-30 in Form von doppelten Notenwerten, wodurch sich sein Notentext

Notenbeispiel 1

a

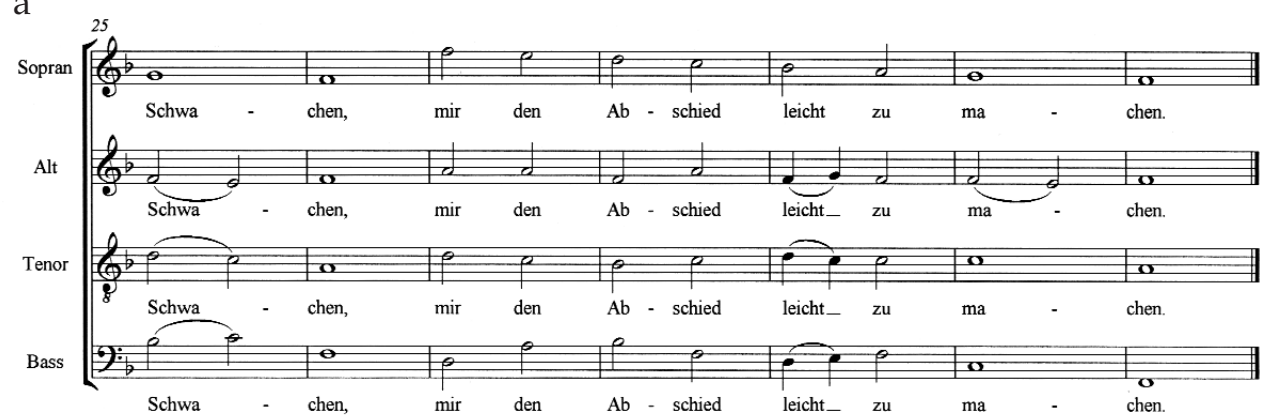

$\mathrm{b}$

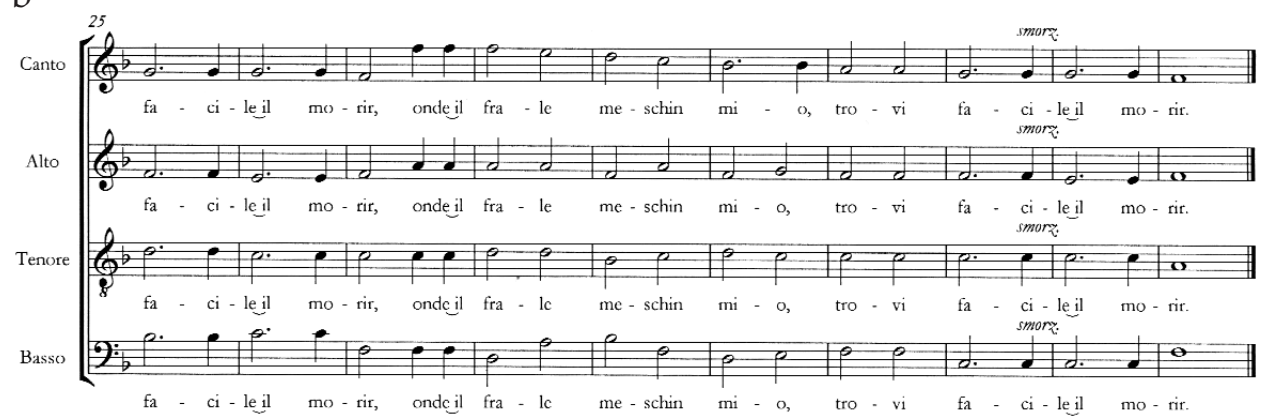


um drei Takte verlängert. Außerdem erhöht Santini den Ausdruck des affektgeladenen Textes

16 onde il frale meschin mio trovi facile il morir.

[damit meine armselige Brust es als leicht empfindet zu sterben.]

durch das Hinzufügen der Vortragsbezeichnung ,,smorz.[ando]“50.

Ein weiterer ästhetisch motivierter Eingriff ist in Chor Nr. 10 Unsere Seele ist gebeuget zu beobachten. Hier wird die musikalische Struktur Grauns verändert, indem Santini den Stimmeneinsatz um zwei Zählzeiten nach vorne zieht. Dadurch wird Grauns synkopische Struktur zu einer auftaktigen umgestaltet. Damit verbunden ist eine Vereinheitlichung der Stimmen. Während bei Graun Sopran und Tenor ab T. 4 durch den Rhythmus Halbenote + Viertelnote festgelegt sind, führen die Alt- und Bass-Stimme den Rhythmus von T. 3 in Viertelnoten fort. Santinis Eingriff besteht nun darin, dass er die rhythmische Gestalt der Sopran- und Tenor-Stimme als vereinheitlichendes Element verarbeitet (siehe Notenbeispiel 2a und b). Dadurch wird Grauns synkopische Struktur in eine auftaktige umgestaltet; dieser Abschnitt erfährt somit eine metrische Veränderung. Dieses Beispiel erinnert an den Eingriff Santinis in seiner Bearbeitung der Bachschen Johannes-Passion, auf den Smend und Fellerer in ihren Studien hinweisen $^{51}$. Im Eingangschor hat Santini die durch Viertelpausen getrennten Rufe "Herr, Herr, Herr" durch eine durchgehende Gesangslinie ersetzt. Während Fellerer das „Bestreben einer ausgeglichenen Deklamation und Rhythmik an Stelle ausdrucksbestimmter Affektrhythmen ${ }^{\prime \prime 52}$ feststellt, zeigt sich für Smend „eine Milderung des Ausdrucks; zugleich aber eine Erhöhung der Gesanglichkeit und eine Steigerung des Gewichtes auf Seiten des Vokalpartes “53. Diese Charakteristika - Vermeidung "ausdrucksbestimmter Affektrhythmen“ zur „Erhöhung der Gesanglichkeit" - zeigen sich auch in unserem Beispiel.

Bei den Chören ist weiterhin zu beobachten, dass Santini Graunsche Melismen durch Textunterlegung teilweise oder ganz aufhebt, während der Notentext unangetastet bleibt. In den Arien begründet sich die Aufhebung jedoch nicht immer durch unterschiedliche Silbenzahl, sondern dient als markante Betonung des jeweiligen - teilweise mehrfach wiederholten - Textes. Beispiele hierfür sind etwa "suo Nume tutelar" ("sein Schutzgott") aus Arie Nr. 4 (siehe Notenbeispiel 3), "già molli tien le gote" (",bald weint aus euch der Schmerz") aus Arie Nr. 9 (siehe Notenbeispiel 4) und "gran vendetta è per me il perdonar" („,euch verzeihn ist meine Rache") aus Arie Nr. 17 (siehe Notenbeispiel 5). In Arie Nr. 19 Singt dem göttlichen Propheten greift Santini ab T. 47 in die musikalische Struktur

\footnotetext{
50 Siehe auch oben Abbildung 1.

51 Vgl. Smend, S. 95 f.; Fellerer, Bachs Johannes-Passion in der lateinischen Fassung Fortunato Santinis, S. 140.

52 Ebd.

53 Smend, S. 96.
} 
Notenbeispiel 2

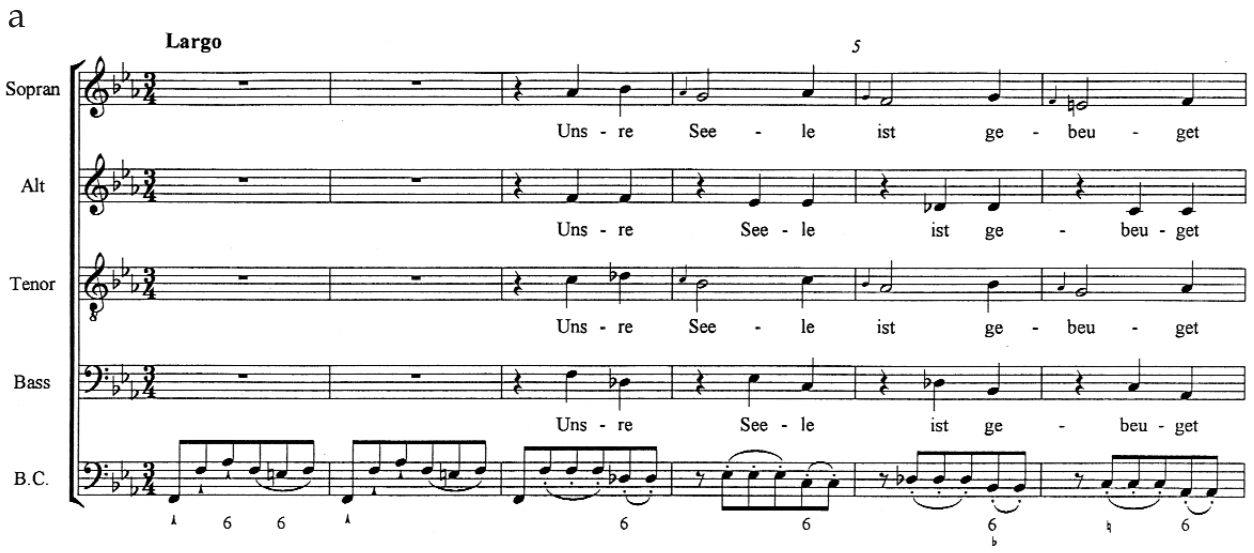

b

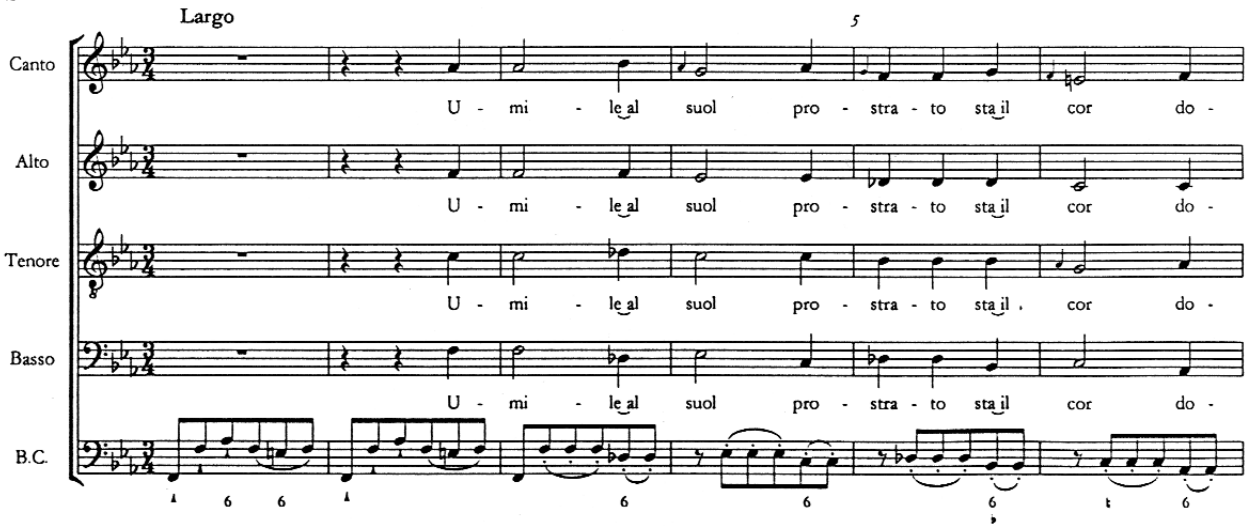

Notenbeispiel 3

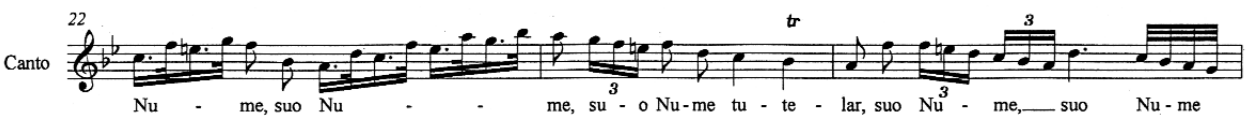

Notenbeispiel 4

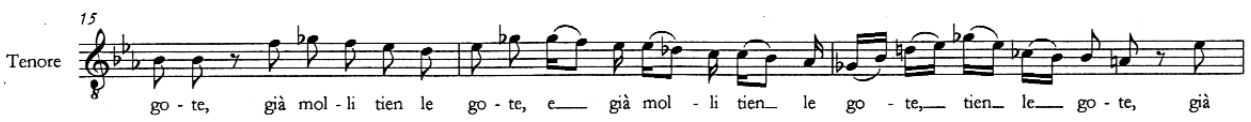

Notenbeispiel 5

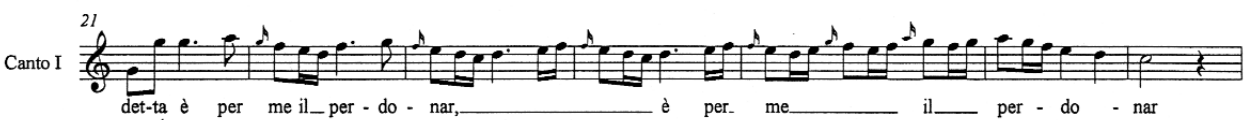


Notenbeispiel 6

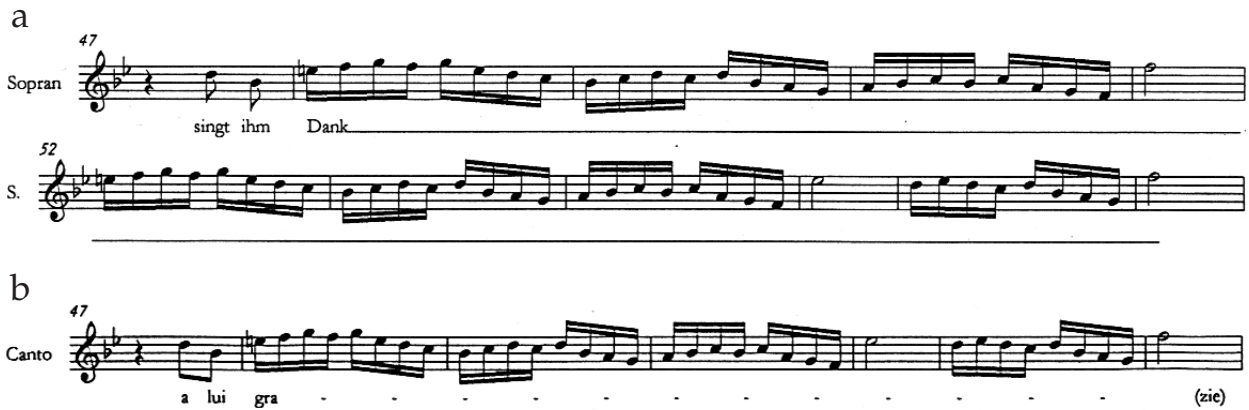

ein, indem er zwar das Graunsche Melisma nachahmt, es aber durch Auslassung der Takte 51-54 verkürzt (siehe Notenbeispiel 6a und b). Außerdem sind die Takte 173-176 deutlich verändert (siehe Notenbeispiel 7a und b). Er verzichtet auf das Alternieren der Gesangsstimme mit den Violinen, eliminiert die Pausen und fährt dann mit Unisonoführung der Oberstimmenmelodie fort, wodurch ein Takt wegfällt. Dieser Eingriff bestätigt die oben beschriebene Präferenz einer fließenden Gesangslinie.

Die Arie Nr. 7 Ein Gebet um neue Stärke ist weniger von Eingriffen in die musikalische Struktur geprägt als von einer gegenüber Graun unstimmigen Textunterlegung. Unstimmig insofern, als Santini für die Arienteile $A+A^{\prime}$ nicht nur die Übersetzung des entsprechenden Graunschen Textes, sondern auch die der ersten Hälfte des Graunschen Textes für den B-Teil verwendet. Dafür nimmt er im B-Teil lediglich die Übersetzung der zweiten Hälfte des Graunschen B-Teils:

A / $\mathrm{A}^{\prime}$

Ein Gebet um neue Stärke

Zur Vollendung edler Werke

Theilt die Wolken, dringt zum Herrn, Und der Herr erhört es gern.

B

Klimm ich zu der Tugend Tempel

Matt den steilen Pfad hinauf:

O so sporn' ich meinen Lauf,

Nach der Wanderer Exempel,

Durch die Hoffnung iener schönen

Über mir erhabenen Scenen,

Und erleichtre meinen Gang

Mit Gebet und Gesang.

\section{$\mathrm{A} / \mathrm{A}^{\prime}$}

Poichè il caldo pregar santo

e d'alte opre eccitatore,

fende i venti e vassi accanto

soavissimo al Signore.

Di virtù se al tempio ascendo

stanco all' arduo sentier.

Vieppiù allor [1.] il corso io stendo [2.]

il passo affretto

imitando il passagier

B

Con la spene di più amene

dolci scene da ammirar, pronto il piede più addiviene fra l'orare ed il cantar 
Notenbeispiel 7

a

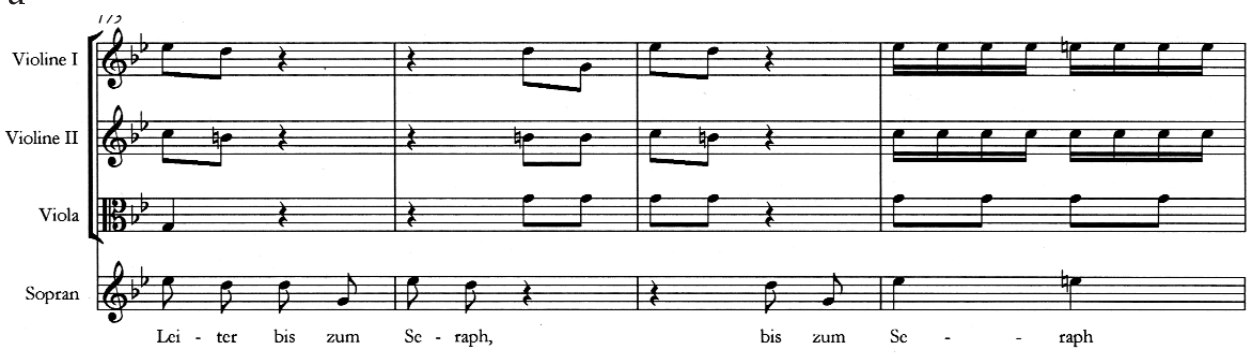

b

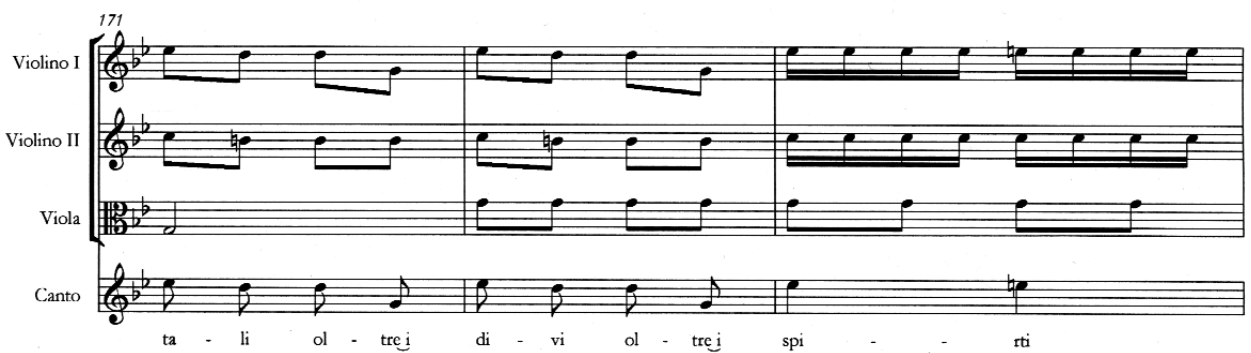

Ob diese Verschiebung ein bewusster Eingriff oder ein Versehen war, ist ungewiss, zumal sie in der Santinischen Bearbeitung ein Einzelfall bleibt. In dieser Arie verkürzt Santini außerdem die Instrumentalüberleitung zum B-Teil von 16 Takten auf 6 Takte, indem er vom 4 . Takt in den 15. Takt dieser Überleitung springt.

Mit Blick auf das Wort-Ton-Verhältnis gestaltet sich die Bearbeitung der Graunschen Rezitative schwieriger als die bisher dargestellten Satzteile. Smend weist auf diese Problematik wie folgt hin ${ }^{54}$ :

„bei den Rezitativen ist vielmehr der Zusammenhang von Wort und Ton so eng, beide sind völlig eine Einheit, daß die Unterlegung neuer Worte so gut wie unmöglich erscheint."

In der Tat erscheinen die bearbeiteten Rezitative Santinis modifiziert. Es stellt sich jedoch die Frage, in welchem Maße sich diese Modifikationen auswirken. Wird der originäre Charakter der Rezitative trotz sprachlicher Differenzen aufrecht erhalten oder verselbstständigt sich die Struktur infolge einer freien melodisch-rhythmischen Bearbeitung? Ohne auf einzelne Beispiele einzugehen, ist der Anteil der Eingriffe in den Rezitativen vergleichsweise gering. Dies resultiert aus dem steten Versuch Santinis, die melodischen Linien der Graunschen Rezitative nachzuzeichnen. In der rhythmischen Gestaltung des Noten-

54 Ebd., S. 92. 
textes entstehen dadurch zwar sprachlich bedingte Modifikationen, aber keine die Kernstruktur betreffenden.

Zusammenfassend lässt sich festhalten, dass Santinis Bearbeitung von einer gewissen Ambivalenz geprägt ist: grundsätzliche Nähe zum Original bei gleichzeitiger Modifikation. Bereits bei der Textanalyse hat sich aufgrund der hohen Anzahl wörtlicher und sinngemäßer Übersetzungen die Nähe zum Originaltext gezeigt. Dies spiegelt sich entsprechend im Großteil der musikalischen Bearbeitung wider. Es gibt zunächst keine großen strukturellen Modifikationen in Besetzung und Abfolge. Darüber hinaus wurden weite Teile des Notentextes nicht verändert oder nur geringfügig modifiziert. In den meisten Fällen sind es rhythmische Änderungen, die in der deklamatorischen Differenz der Einzelsprachen begründet sind. Dies ist in den Chorälen und besonders in den Rezitativen der Fall, in welchen Santini fast durchgehend die Originalgestalt des Notentextes modifiziert und doch so nah wie möglich die ursprünglichen Melodielinien verfolgt.

Dass Santini jedoch auch durchaus mit freien - das heißt nicht sprachlich bedingten - Modifikationen arbeitet, erweist sich aus den besonders in den Chören und Arien gezeigten Eingriffen, wie etwa der Verkürzung instrumentaler Vor- und Zwischenspiele, der freien Handhabung Graunscher Melismen oder der vereinheitlichenden Tendenz des Materials zugunsten einer fließenden Gesangslinie.

Aus der Analyse kann man darüber hinaus auf Santinis Arbeitsweise schließen: Er fertigte zunächst eine Übersetzung an, die zwar im Allgemeinen dem deutschen Text folgt, jedoch in eine eigenständige lyrische Form gebracht und mit sprachlichen Modifikationen versehen wurde. Erst in einem zweiten Arbeitsschritt passte er den Notentext seiner Übersetzung an.

\section{Schlussbetrachtung und Bewertung}

Konfessionsübergreifende Bearbeitungen wie Santinis La Morte di Gesù waren im 18. Jahrhundert nicht unüblich. Ein prominentes Beispiel hierfür ist das Stabat Mater von Giovanni Battista Pergolesi, das vor allem in Deutschland seit Johann Sebastian Bachs Bearbeitung unter dem Titel Tilge, Höchster, meine Sünden (BWV 1083/243a) eine enorme Rezeption erfuhr. Für die Rezeption in Deutschland waren deutsche Neutextierungen und Eingriffe in die musikalische Gestalt maßgeblich, was nicht selten auf kritischen Äußerungen hinsichtlich der kompositorischen Faktur beruhte. Die Bandbreite der Neutextierungen des genuin katholischen Textes reichte von wörtlichen Übertragungen - wie etwa bei Christoph Martin Wieland - bis hin zur "Adaption durch Entkatholisie- 
rung des Textes ${ } 55$ für eine protestantische Musikpraxis. Für Letzteres steht paradigmatisch Friedrich Gottlieb Klopstocks deutsche Parodie ${ }^{56}$.

Johann Adam Hiller, der im norddeutschen Raum zahlreiche ältere Werke aufführte, griff bei seiner Bearbeitung des Stabat Mater auf eben diese Klopstocksche Parodie zurück und äußert sich im Vorbericht seiner Druckausgabe von 1776 folgendermaßen ${ }^{57}$ :

„Der Fleiß so manches berühmten und braven Componisten, der täglich neue Stücke, in allerley Art, hervorbringt, ist allerdings zu loben. Wenn wir aber alles vorhergegangene Gute darüber ganz vergessen; wenn wir die kleine Mühe scheuen, ihm diejenige Gestalt zu geben, die es nach unseren Verfassungen haben soll, so handeln wir ungerecht."

Die angesprochenen zeitgemäßen Veränderungen der älteren Werke betreffen Kürzungen des Gesamtumfangs, indem Hiller Nummern kürzt, sie austauscht oder Streichungen innerhalb der Stücke vollzieht. Während vor allem aufführungspraktische Gründe Hillers Modernisierung der Instrumentation erklären, sind darüber hinaus auch Eingriffe in harmonische und melodische Zusammenhänge festzustellen ${ }^{58}$.

Durch den spätestens im 19. Jahrhundert aufkommenden Historismus entwickelte sich allmählich eine den originalen Notentext als verbindlich betrachtende Sichtweise. Dass dies jedoch erst im späten 19. Jahrhundert endgültig griff, belegt etwa Felix Mendelssohn Bartholdys berühmte Berliner Wiederaufführung der Johann Sebastian Bachschen Matthäus-Passion von 1829. Ein Blick auf die hierfür bearbeitete Fassung zeigt, dass Mendelssohn durchaus in die Bachsche Partitur eingreift. Am augenscheinlichsten ist die Reduzierung auf lediglich 35 Nummern. Zudem gibt es Änderungen und Ergänzungen in Fragen der Besetzung und des Notentextes, welche jedoch, verglichen mit der Hillerschen Bearbeitungspraxis des späten 18. Jahrhunderts, deutlich geringer ausfallen ${ }^{59}$.

Wie kann man nun Santinis Bearbeitung des Tod Jesu in den historischen Kontext einordnen und bewerten? Zunächst weist Santinis italienischer Text keinerlei Tendenzen einer ,Entprotestantisierung' auf - eine Konfessionsangleichung nach Klopstockschem Vorbild findet nicht statt. Die Modifikationen basieren vielmehr auf der Ebene einer eigenen lyrischen Form und einer af-

55 J. Heidrich, Protestantische Kirchenmusikanschauung in der zweiten Hälfte des 18. Jahrhunderts. Studien zur Ideengeschichte ,wahrer' Kirchenmusik, Göttingen 2001 (= Abhandlungen zur Musikgeschichte 7), S. 68.

${ }^{56}$ Vgl. M. Marx-Weber, Parodie als Beispiel dichterischer Anpassung. Klopstocks deutscher Text zu Pergolesis "Stabat mater", in: Studien zum deutschen weltlichen Kunstlied des 17. und 18. Jahrhunderts, hrsg. v. G. Busch u. a., Amsterdam u. a. 1992, S. 269-290.

57 Johann Baptist Pergolese vollständige Passionsmusik zum Stabat Mater, mit der Klopstockischen Parodie; in der Harmonie verbessert, mit Oboen und Flöten verstärckt, und auf vier Stimmen gebracht von Johann Adam Hiller, Leipzig 1776.

58 Vgl. ferner Marx-Weber, Johann Adam Hillers Bearbeitung von Händels „Messias“, in: Händel-Jahrbuch 39 (1993), S. 60-77.

59 Vgl. M. Geck, Die Wiederentdeckung der Matthäuspassion im 19. Jahrhundert. Die zeitgenössischen Dokumente und ihre ideengeschichtliche Deutung, Regensburg 1967 (= Studien zur Musikgeschichte des 19. Jahrhunderts 9). 
fektgeladenen Sprache, so dass Santinis Text als relativ genaue Übertragung angesehen werden kann. Ähnlich verhält es sich mit seiner musikalischen Bearbeitung, die grundsätzlich der Graunschen Anlage folgt und innerhalb der musikalischen Teile nur partiell verändert wird. Allerdings sind Santinis Eingriffe verglichen mit der zeitgenössischen Bearbeitungspraxis von einem eher geringen Ausmaß.

Entscheidend für Santinis Konzeption war das Streben, alte Musik in einer relativ ursprünglichen Form zu erhalten und bekannt zu machen. Für dieses Vorhaben war der Standort Rom offenbar ungeeignet, da es gerade in den konservativ-kirchenmusikalischen Kreisen Roms kaum Aufführungsmöglichkeiten gab, insbesondere nicht für eine italienische Bearbeitung des Graunschen Tod Jesu mit einem protestantisch fundierten Text. Die von Mendelssohn erwähnte positive Rückmeldung aus Neapel lässt darauf schließen, dass den Bearbeitungen Santinis dort größerer Erfolg beschieden war. Langfristig konnten sie sich jedoch auch dort nicht etablieren. Die Rezeption deutscher Musik in Italien fand insgesamt nur begrenzt statt, da es grundsätzliche Vorbehalte gegenüber einer deutschen Stilistik gab und man sich von einer als philosophisch und akademisch geltenden Musik nicht ,eindeutschen' lassen wollte ${ }^{60}$. Vor diesem Hintergrund ist Santinis kosmopolitische Offenheit bemerkenswert. Die Beschäftigung mit fremdsprachiger Musik - über konfessionelle Barrieren hinweg - belegt sein Interesse am Kunstwerk, fern ab von einer ihm bisweilen vorgeworfenen Sammelwut. Die häufigen Vermerke auf den bearbeiteten Handschriften wie etwa "per far conoscere il merito" belegen sein vorbehaltloses Bemühen, die Werke für Italien einzurichten. In einem Brief Santinis an Gaetano Gaspari im Zusammenhang mit der Bachschen Matthäus-Passion betont er dies folgendermaßen: „, . . ma disgrazia per gl'Italiani, essendo il testo in tedesco. ${ }^{161}$ Santinis relativ getreue Bearbeitung des Graunschen Tod Jesu ist zwar im Italien des 19. Jahrhunderts nicht zu etablieren gewesen, erhält jedoch aus der Retrospektive eine Sonderstellung in der Rezeption deutscher geistlicher Musik im frühen 19. Jahrhundert.

${ }^{60}$ Vgl. hierzu Tagliavini, S. 301; außerdem die Correspondenz aus Rom, in: Allgemeine musikalische Zeitung 48 (1846), Sp. 780-782.

61 „Schade aber für die Italiener, dass der Text auf deutsch ist.“ Brief vom 21.3.1858 (zitiert nach Tagliavini, S. 307). 